\title{
Detector Backgrounds for a High Energy Muon Collider
}

\author{
O. Benary \\ Tel-Aviv University, Israel \\ and \\ S. Kahn, I. Stumer \\ Brookhaven National Laboratory
}

January 2000 


\title{
Detector Backgrounds for a High Energy Muon Collider
}

\author{
O. Benary ${ }^{*}$ S. Kahn ${ }^{\S}$, and I. Stumer ${ }^{\S}$ \\ ${ }^{\S}$ Brookhaven National Laboratory, Upton, NY 11973 \\ *Tel-Aviv University, Ramat-Aviv, Tel-Aviv 69978, Isreal
}

\begin{abstract}
The potentially large background produced from the decay products of muons could affect the quality of the physics in a high energy muon collider. This paper examines the kinds of background present and their expected rate. The results are based on a simulation of muon colliders with center of mass energies of $100 \mathrm{GeV}, 500 \mathrm{GeV}$ and $4 \mathrm{TeV}$.
\end{abstract}

\section{INTRODUCTION}

How well the background can be controlled will determine the kind of physics that can be done in a muon collider. Most backgrounds are associated with the products of the decaying muons that get into the detector region. A $2 \mathrm{TeV} / \mathrm{c}$ muon beam with $2 \times 10^{12} \mu$ per bunch will produce $2 \times 10^{5}$ decays per meter. The number of decays per length scales with $1 / \gamma$ because of the Lorentz contraction. The electrons produced from muon decays will not have the designed momentum of the collider ring and will either interact with the wall of the beam chamber producing electromagnetic showers or produce bremsstrahlung in regions of large transverse magnetic field. The design of a detector for a muon collider will be constrained by the necessity to reduce the electromagnetic background.

Detector backgrounds have been studied for a potential muon collider at three different energies:

- A $50 \times 50 \mathrm{GeV} / \mathrm{c}$ Higgs factory collider.

- A $250 \times 250 \mathrm{GeV} / \mathrm{c}$ muon collider at the same energy as the phase 1 NLC.

- A $2 \times 2 \mathrm{TeV} / \mathrm{c}$ muon collider at an energy not accessible to an electron collider.

Results of these studies have been reported elsewhere [1-2]. No explicit investigation has been done for backgrounds in muon colliders with $\sqrt{s}$ between $10 \mathrm{TeV}$ and 100 $\mathrm{TeV}$. This paper will review what has been learned about muon collider background in the studies that have been previously performed. Extrapolation to higher energies may not be straight forward since the shielding geometry and the beam final focus elements have been optimized at each energy to reduce backgrounds. The effort to minimize the backgrounds will have a strong influence on both the design of the detector and the design of the magnets in the final focus of the intersection region (IR). 


\section{BACKGROUNDS}

The following classes of backgrounds have been investigated for a muon collider:

- Muon Decay Background.

- Beam Halo Background.

- Beam-Beam Interactions.

Most of the work to date has been devoted to the backgrounds associated to muon decays. The backgrounds associated to muon decays include:

- Electron showers.

- Synchrotron Radiation and pair production.

- Photonuclear interactions producing hadrons.

- Bethe-Heitler muon production.

These will be discussed thoroughly in the following sections. Beam halo backgrounds come from accelerator sources. The beam scraping system will be designed to reduce halo, however even a small residual muon halo can produce a large background in the detector.

The backgrounds associated with the muon decay have been studied with a GEANT simulation of a detector that would be appropriate for a muon collider. Figure 1 shows a sketch of the geometry of such a detector along with the final focus magnets of the collider ring. The detector geometry includes the following features:

- Conical tungsten shield over the beam extending to $20^{\circ}$ in both forward and backward directions. The shield prevents most of the electromagnetic showers from penetrating into the detector region.

- Expanding shield inner cone beyond minimum aperture position (1.1 meters from IP for $2 \times 2 \mathrm{TeV}$ collider) is $4 \sigma$ of the beam size.

- Between IP and minimum aperture point the inner radius is an inverse cone with an angle equal to but opposite sign of the previously mentioned angle of expansion after the minimum aperture point. These cones are designed so that the detector does not see any surface where incident decay electrons could have interacted.

- The open space between the IP and the beginning of the tungsten shield is small. It is only $3 \mathrm{~cm}$ for the $2 \times 2 \mathrm{TeV}$ collider.

- The inner surface of each shield is shaped in a sawtooth manner. These steps and slopes collimate the electrons in the beam and maximize the absorption of the electromagnetic showers from the electrons that graze the cone surface. This reduces the funneling of low energy electrons down the beam pipe. Figure 2 shows the geometry of the inner surface of the tungsten shield.

- An $8 \mathrm{~T}$ dipole magnet with collimator inside is placed upstream of the first quadrupole magnet to sweep decay electrons away before the final collimation. 
Table 1: Parameters describing the geometry of the intersection region for the muon colliders studied at three energies.

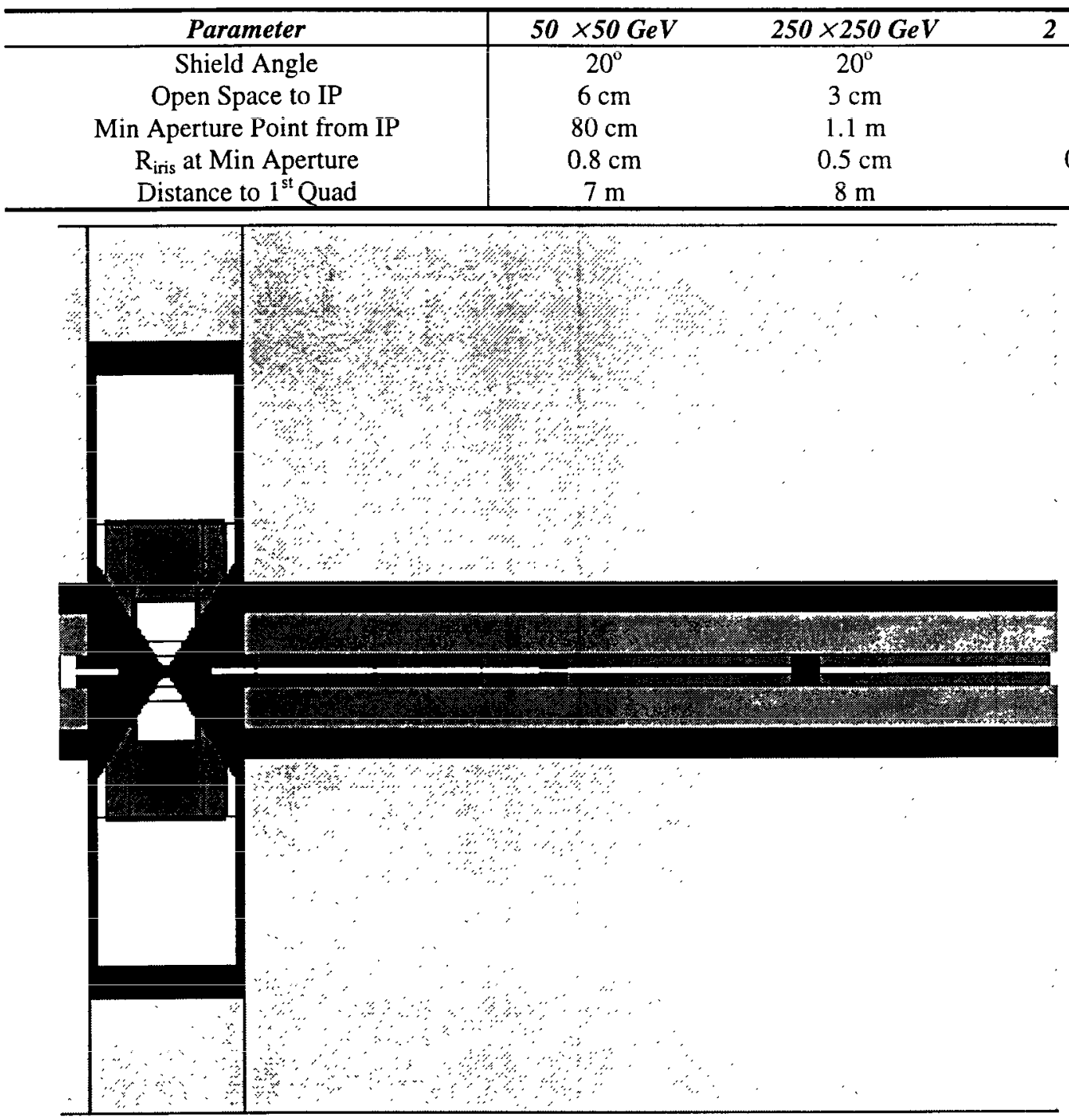

FIGURE 1. Sketch of the IP region and 130 meters of the final focus magnet system. This sketch shows the geometry of the detector used in the GEANT simulation of the $2 \times 2 \mathrm{TeV}$ Muon Collider.

\section{Muon Decay Background}

The electromagnetic showers produced from the muon decay electrons interacting with the beam chamber wall are mostly contained in the tungsten shielding. A small residual flux of soft photons does reach the detector region. Electrons in the showers are trapped at small radius by the $2-4 \mathrm{~T}$ detector solenoid field. This is shown in table 2. Table 2 also shows fluences for protons, neutrons, and pions. Hadrons are produced in $\sim 1 \%$ of the interactions in an electromagnetic shower. These hadrons are produced as a result of photonuclear processes:

- Giant Dipole Resonance in the energy region $5<\mathrm{E}_{\gamma}<30 \mathrm{MeV}$. 
- Quasi-Deuteron Region in the energy region $30<\mathrm{E}_{\gamma}<30 \mathrm{MeV}$.

- Baryon Resonance Production in the energy region $150 \mathrm{MeV}<\mathrm{E}_{\gamma}<2 \mathrm{GeV}$.

- Vector Dominance in the energy region $\mathrm{E}_{\gamma}>2 \mathrm{GeV}$.

The GEANT program used in these simulations has been modified to include these processes. Hadron production is also possible at the highest energies through leptoproduction. This process has been ignored in the studies so far, however they certainly would be important at very high energy muon colliders. The MICAP option in GEANT for hadron showering package was used since it handles neutrons down to thermal energies. Low energy neutrons can be bothersome since they live a long time. They can bounce around entering the same detector element several times giving extraneous signals. In order to alleviate this problem, borated-polyethelene is placed where possible around the tungsten shielding and the calorimeter to soak up as many neutrons as possible. This reduces the neutron flux substantially.

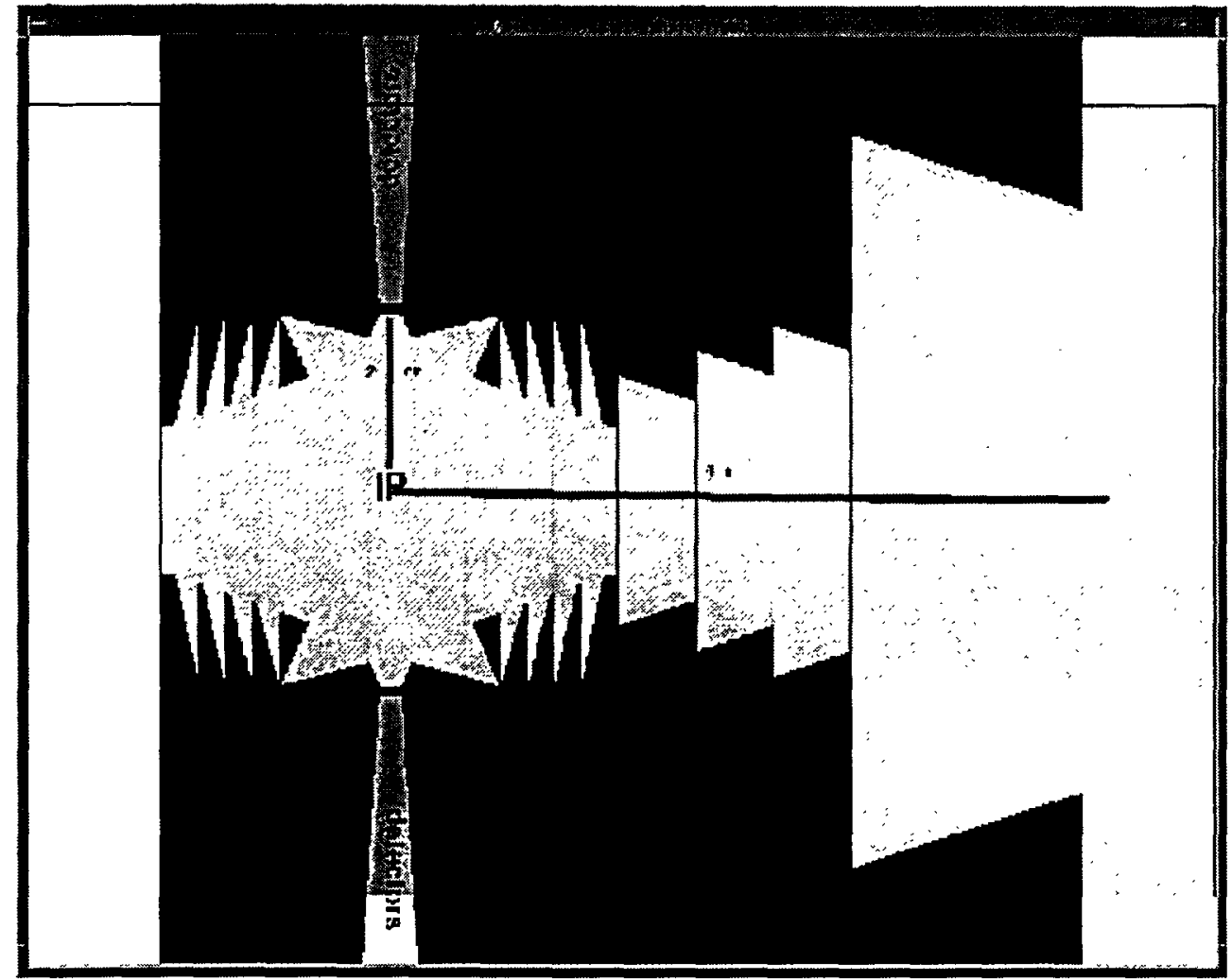

FIGURE 2: Absorber and collimation system on the inside surface of the tungsten shield. This system is designed to reduce electrons in the beam pipe in the vicinity of the IP.

Shown in Table 2a, b are the radial particle fluxes in $\mathrm{cm}^{-2} /$ crossing for two bunches of $10^{12} \mu$ each at different radii for the $50 \times 50 \mathrm{GeV}$ and $2 \times 2 \mathrm{TeV}$ machines. The 
rates are shown for $\gamma, \mathrm{n}, \mathrm{p}, \pi, \mathrm{e}$, and $\mu$. The energy thresholds used to define the particle fluxes are $\mathrm{E}_{\gamma}>25 \mathrm{keV}, \mathrm{E}_{\mathrm{n}}>40 \mathrm{keV}, \mathrm{E}_{\mathrm{p}}>10 \mathrm{MeV}, \mathrm{E}_{\pi}>10 \mathrm{MeV}$. The fluxes between $5 \mathrm{~cm}$ and $20 \mathrm{~cm}$ are what a vertex detector would see. A central tracker might be located at $50-100 \mathrm{~cm}$ and the calorimeter would start at $150 \mathrm{~cm}$. The fluxes shown in Table 2 can be interpreted as occupancy in a $300 \mu \mathrm{m} \times 300 \mu \mathrm{m}$ silicon pad detector that might be used as a vertex detector. If one assumes that the probability that low energy photons and neutrons interact in the silicon pad detector is 0.003 and 0.0001 , respectively, hits and occupancy rates can be calculated. Table $3 \mathrm{~A}$ and $\mathrm{B}$ show the background hits and occupancy for the $50 \times 50 \mathrm{GeV}$ and $2 \times 2 \mathrm{TeV}$ colliders, respectively. Figure 3 shows the occupancy for all three energies studied. The lefthand part of Figure 3 shows the occupancies for all particles while the right-hand part shows the occupancies for the charge particles only. The total occupancy is above $1 \%$ at small radii, mostly due to photon conversions. The charge particle occupancy is equal or below $1 \%$ at all the radii in the figure. One can reduce the occupancy rates by using smaller pixel sizes or using new innovated detector ideas.

TABLE 2A. Flux per crossing for two $50 \mathrm{GeV}$ beams of $10^{12} \mu$ per bunch.

\begin{tabular}{c|cccccc} 
Radius & $\gamma$ & $\mathbf{n}$ & $\mathbf{p}$ & $\boldsymbol{\pi}$ & $\mathbf{e}$ & $\mu$ \\
\hline $\mathbf{5} \mathbf{~ c m}$ & 4300 & 32 & & & 3.8 & 0.15 \\
$\mathbf{1 0} \mathbf{~ c m}$ & 1100 & 36 & & 0.24 & 0.3 & 0.07 \\
$\mathbf{1 5} \mathbf{~ c m}$ & 480 & 75 & & 0.11 & & 0.03 \\
$\mathbf{2 0} \mathbf{~ c m}$ & 270 & 98 & & 0.09 & 0.007 \\
$\mathbf{5 0} \mathbf{~ c m}$ & 40 & 37 & 0.05 & 0.015 & 0.0004 \\
$\mathbf{1 0 0} \mathbf{~ c m}$ & 9 & 18 & 0.005 & & 0.0002 \\
$\mathbf{1 5 0} \mathbf{~ c m}$ & 4 & 9 & 0.02 & & $2.1 \times 10^{-5}$
\end{tabular}

TABLE 2B. Flux per crossing for two $2 \mathrm{TeV}$ beams of $10^{12} \mu$ per bunch.

\begin{tabular}{c|cccccc} 
Radius & $\gamma$ & $\mathbf{n}$ & $\mathbf{p}$ & $\boldsymbol{\pi}$ & $\mathbf{e}$ & $\mu$ \\
\hline $\mathbf{5} \mathbf{~ c m}$ & 2700 & 120 & 0.05 & 0.9 & 2.3 & 1.7 \\
$\mathbf{1 0} \mathbf{~ c m}$ & 750 & 110 & 0.20 & 0.4 & - & 0.7 \\
$\mathbf{1 5} \mathbf{~ c m}$ & 350 & 100 & 0.13 & 0.4 & - & 0.4 \\
$\mathbf{2 0} \mathbf{~ c m}$ & 210 & 100 & 0.13 & 0.3 & - & 0.1 \\
$\mathbf{5 0} \mathbf{~ c m}$ & 70 & 120 & 0.08 & 0.05 & - & 0.02 \\
$\mathbf{1 0 0} \mathbf{~ c m}$ & 31 & 50 & 0.04 & 0.003 & - & 0.008 \\
$\mathbf{1 5 0} \mathbf{~ c m}$ & & & & & & 0.003
\end{tabular}

TABLE 3A. Background hits and occupancy for $50 \times 50 \mathrm{GeV} \mu$ collider. Hit rates are quoted for two beams with bunches of $10^{12} \mu$ 's each. Occupancy is quoted for two beams with $4 \times 10^{12} \mu$ 's each and that the silicon detector has $300 \times 300 \mu \mathrm{m}$ pads.

\begin{tabular}{c|cccccc}
$\begin{array}{c}\text { Radius } \\
\mathbf{c m}\end{array}$ & $\begin{array}{c}\gamma \\
\mathbf{c m}^{-2}\end{array}$ & $\begin{array}{c}\mathbf{n} \\
\mathbf{c m}^{-2}\end{array}$ & $\begin{array}{c}\text { Charged } \\
\mathbf{c m}^{-2}\end{array}$ & $\begin{array}{c}\text { Hits } \\
\mathbf{c m}^{-2}\end{array}$ & $\begin{array}{c}\text { Total } \\
\text { Occupancy }\end{array}$ & $\begin{array}{c}\text { Charged } \\
\text { Occupancy }\end{array}$ \\
\hline $\mathbf{5}$ & 13 & 0.03 & 4 & 17 & $6.1 \%$ & $1.4 \%$ \\
$\mathbf{1 0}$ & 3.3 & 0.04 & 0.6 & 4 & $1.4 \%$ & $0.2 \%$ \\
$\mathbf{1 5}$ & 1.4 & 0.07 & 0.14 & 1.6 & $0.6 \%$ & $0.05 \%$ \\
$\mathbf{2 0}$ & 0.8 & 0.1 & 0.10 & 1.0 & $0.4 \%$ & $0.04 \%$ \\
$\mathbf{5 0}$ & 0.1 & 0.03 & 0.065 & 0.2 & $0.07 \%$ & $0.02 \%$ \\
$\mathbf{1 0 0}$ & 0.03 & 0.02 & 0.005 & 0.06 & $0.02 \%$ & $0.002 \%$ \\
$\mathbf{1 5 0}$ & 0.01 & 0.01 & 0.02 & 0.04 & $0.014 \%$ & $0.007 \%$
\end{tabular}


TABLE 3B. Background hits and occupancy for $2 \times 2 \mathrm{TeV} \mu$ collider. Hit rates are quoted for two beams with bunches of $10^{12} \mu$ 's each. Occuipancy is quoted for two beams with $2 \times 10^{12} \mu$ 's each and that the silicon detector has $300 \times 300 \mu \mathrm{m}$ pads.

\begin{tabular}{c|cccccc}
$\begin{array}{c}\text { Radius } \\
\mathbf{c m}\end{array}$ & $\begin{array}{c}\gamma \\
\mathbf{c m}^{-2}\end{array}$ & $\begin{array}{c}\mathbf{n} \\
\mathbf{c m}^{-2}\end{array}$ & $\begin{array}{c}\text { charged } \\
\mathbf{c m}^{-2}\end{array}$ & $\begin{array}{c}\text { Hits } \\
\mathbf{c m}^{-2}\end{array}$ & $\begin{array}{c}\text { Total } \\
\text { Occupancy }\end{array}$ & $\begin{array}{c}\text { Charged } \\
\text { Occupancy }\end{array}$ \\
\hline $\mathbf{5}$ & 8.1 & 0.012 & 5.0 & 13.1 & $2.4 \%$ & $0.9 \%$ \\
$\mathbf{1 0}$ & 2.3 & 0.011 & 1.3 & 3.6 & $0.65 \%$ & $0.23 \%$ \\
$\mathbf{1 5}$ & 1.1 & 0.010 & 0.93 & 2.0 & $0.36 \%$ & $0.17 \%$ \\
$\mathbf{2 0}$ & 0.6 & 0.010 & 0.53 & 1.1 & $0.20 \%$ & $0.10 \%$ \\
$\mathbf{5 0}$ & 0.2 & 0.012 & 0.15 & 0.36 & $0.06 \%$ & $0.03 \%$ \\
$\mathbf{1 0 0}$ & 0.1 & 0.005 & 0.05 & 0.16 & $0.03 \%$ & $0.01 \%$
\end{tabular}

The lifetime of a vertex detector in a high radiation environment is of concern. The radiation damage to a silicon vertex detector at $5 \mathrm{~cm}$ from the beam can be estimated. Table 4 shows the hits $/ \mathrm{cm}^{2} / \mathrm{yr}\left(1\right.$ year $\left.=10^{7} \mathrm{sec}\right)$ and expected lifetime of the device under normal operating conditions. An acceptable number of hits for the silicon vertex detector lifetime is $1.5 \times 10^{14}$.

TABLE 4. Radiation damage by neutrons on a silicon vertex detector situated at $5 \mathrm{~cm}$ from the beam. It is assumed that the machine is operating $10^{7}$ sec/year at $15 \mathrm{~Hz}$ with 1000 turns per machine fill. The neutrons counted are those with $E_{\text {kin }}>100 \mathrm{keV}$.

\begin{tabular}{c|cccc}
\multirow{2}{*}{$\begin{array}{c}\text { Energy } \\
\end{array}$} & $\mu /$ /bunch & $\begin{array}{c}\text { Neutrons } / \mathbf{c m}^{2} \\
\text { Per crossing }\end{array}$ & Hits $/ \mathrm{cm}^{2} /$ year & $\begin{array}{c}\text { Lifetime } \\
\text { (years) }\end{array}$ \\
\hline $\mathbf{5 0} \times \mathbf{5 0 ~ G e V}$ & $4 \times 10^{12}$ & 30 & $1.8 \times 10^{13}$ & 8 \\
$\mathbf{2 5 0} \times \mathbf{2 5 0 ~ G e V}$ & $4 \times 10^{12}$ & 50 & $3 \times 10^{13}$ & 5 \\
$\mathbf{2} \times \mathbf{2} \mathbf{~ T e V}$ & $2 \times 10^{12}$ & 100 & $3 \times 10^{13}$ & 5
\end{tabular}
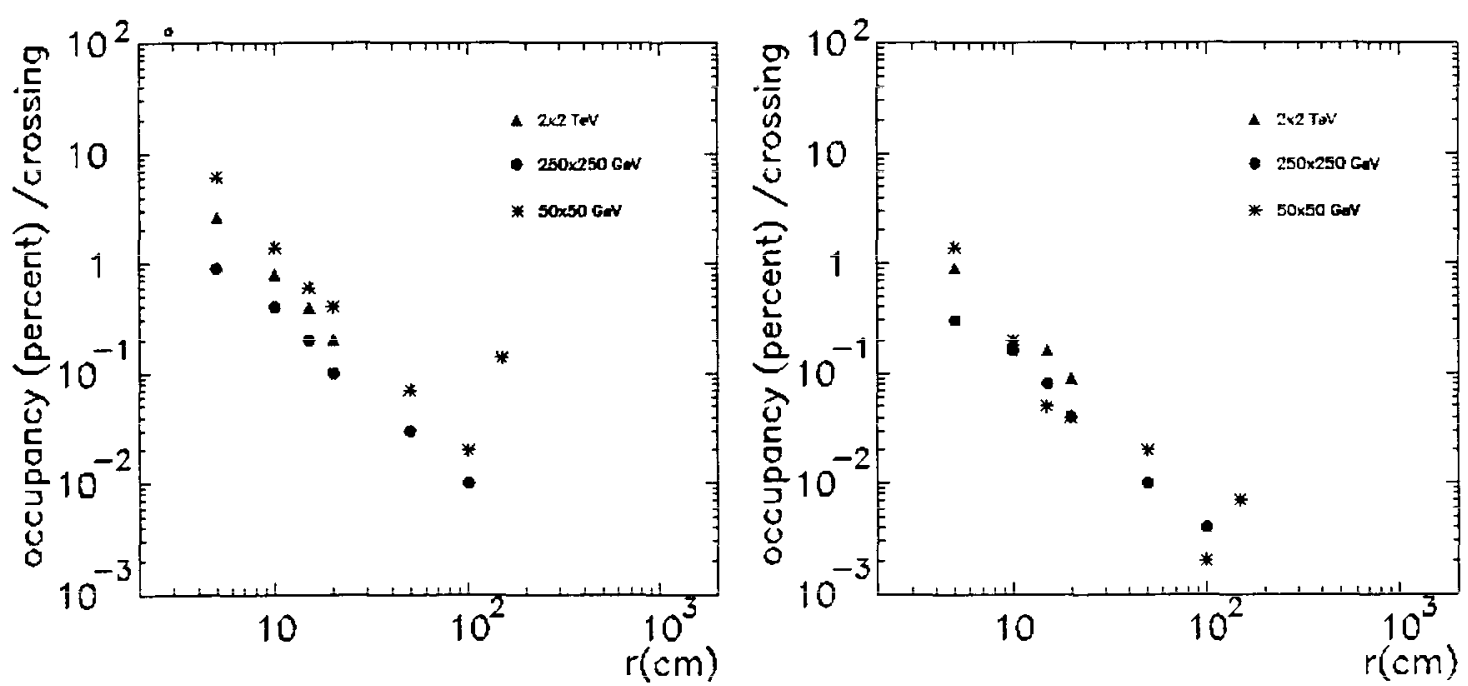

FIGURE 3: Occupancy for $300 \mu \mathrm{m} \times 300 \mu \mathrm{m}$ silicon pads, as a function of the radius for the three energies studied. Left-hand figure shows the total occupancy while the right-hand figure shows the occupancy for charged particles only. 


\section{Bethe-Heitler Muons}

A significant flux of muons with high energy come from $\mu$ pair production in electromagnetic showers. These Bethe-Heitler muons will be a concern at high energy colliders. Figure 4 shows the spectrum of Bethe-Heitler muons in the final focus region for the $2 \times 2 \mathrm{TeV}$ collider. This figure is obtained by convoluting the energy spectrum from the decay of $2 \mathrm{TeV}$ muons $\left.\left(<\mathrm{E}_{\text {eiectron }}\right\rangle=670 \mathrm{GeV}\right)$ with the $\mu$ pair production cross section. Figure 5 displays the trajectories of typical Bethe-Heitler muons from the last $130 \mathrm{~m}$ section of beam pipe before the detector in the $2 \times 2 \mathrm{TeV}$ collider. The most serious effect of these muons is that they may make deeply inelastic collisions in the electromagnetic or hadronic calorimeter. A large deposit of energy in a single cell in the vicinity of other jets can cause severe fluctuations in the global parameters such as transverse energy or missing transverse energy. This is a more serious problem for the higher energy muon colliders.

Table 5 gives the attributes of the Bethe-Heitier muons found at the three energies. In the $250 \times 250 \mathrm{GeV}$ and $2 \times 2 \mathrm{TeV}$ cases there is massive lead shielding around the final focus quadrupoles to reduce the Bethe-Heitler muons as much as possible. In the higher energy cases $\leq 1 \%$ of these muons reach the calorimeter. In the $2 \times 2 \mathrm{TeV}$ case the B-H muons have $\left\langle\mathrm{p}_{\mu}\right\rangle=15.4 \mathrm{GeV} / \mathrm{c}$ and deposit $2.9 \mathrm{GeV}$ in the calorimeter on average. This sits on top of a uniform pedestal of $100 \mathrm{GeV}$ to $100 \mathrm{TeV}$ of deposited energy from all other sources. The size of the pedestal is dependent on the calorimeter technology used. Since calorimeters based on scintillator technology will have a higher pedestal of deposited energy, they may not be a desirable choice. The high neutron background in the calorimeter favors the use of liquid argon as the active calorimeter medium. The $\mathrm{B}-\mathrm{H}$ muons cause large local fluctuations in the energy deposited in a cell. These energy spikes can (1) cause false triggers when event selection is based on transverse or overall energy balance, (2) generate false jets, and (3) give incorrect energy of real jets. False jet generation can be eliminated with longitudinal energy balance. Errors in energy of real jets can be a more serious problem. They can be reduced to some extent with radial energy distribution cuts.

Figure 6 shows the energy deposition for $\mathrm{B}-\mathrm{H}$ muons in calorimeter cells segmented in $\Delta \phi$ and $\Delta \cos \theta$ for the $2 \times 2 \mathrm{TeV}$ muon collider. In this study the lead shield around the final focus quadrupoles is not included. The left-hand figure has no timing cut, where as the right-hand plot has a $1 \mathrm{~ns}$ timing in synch with the particles from the beam IP. The figure shows that B-H muons in the central (barrel) part of the calorimeter are eliminated. Figure 7 illustrates the paths of muons from the IP and $\mathrm{B}-\mathrm{H}$ muons arriving at the central and the forward calorimeters. A B-H muon, which was in time with the muon beam at its production point at $100 \mathrm{~m}$ away, would go directly to the calorimeter, whereas a muon heading to the IP would travel $100 \mathrm{~m}$ plus an additional time for the transit of a particle created at the IP to reach the calorimeter. 


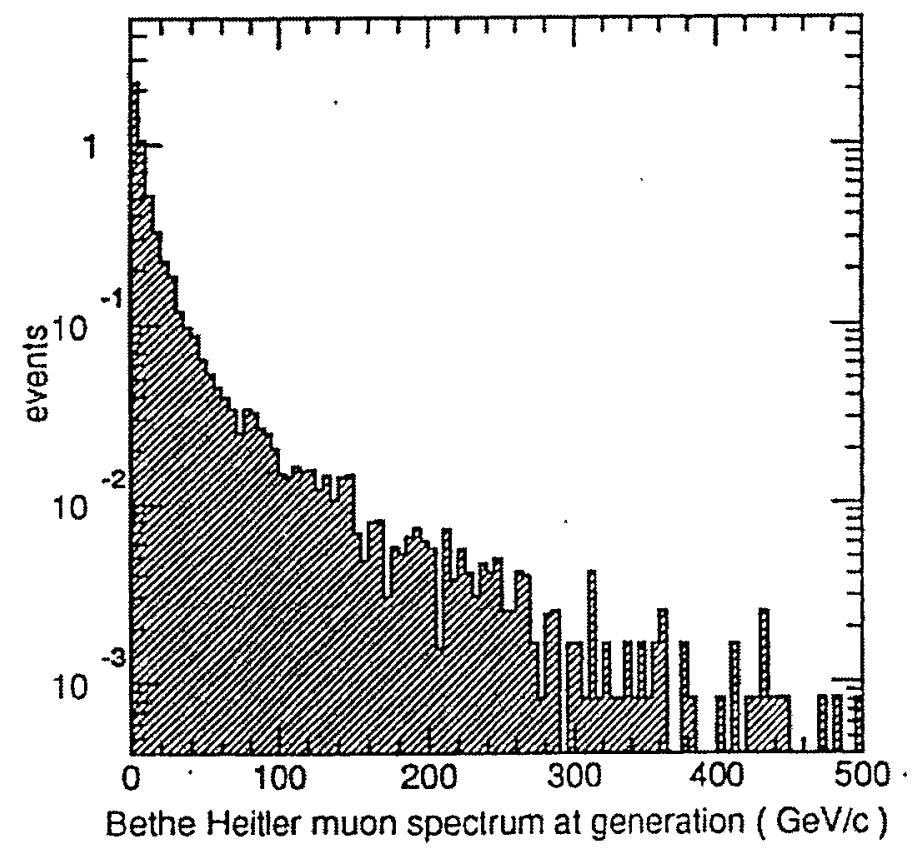

FIGURE 4. Bethe-Heitler muon spectrum at generation.

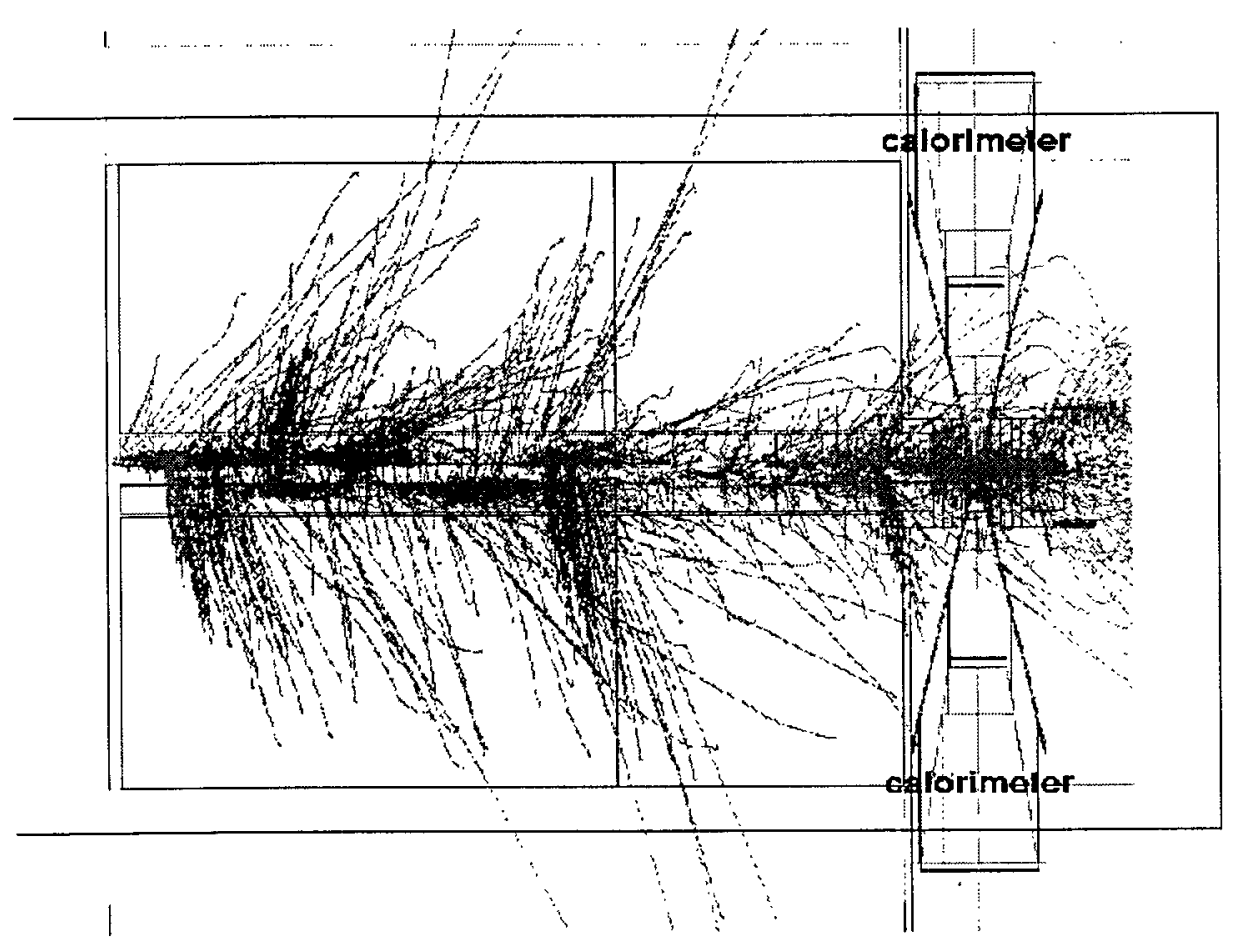

FIGURE 5. Trajectories of Bethe-Heitler muons from sources in the shielding around the beampipe in the last $130 \mathrm{~m}$ from the IP for the $2 \times 2 \mathrm{TeV}$ collider. The outer edge of the calorimeter is approximately $4 \mathrm{~m}$ 
TABLE 5: Attributes of Bethe-Heitler muons at the energies of the three muon colliders studied. This table gives the parameters at the source of the muon pairs and in the calorimeter. It is assumed that lead shielding surrounds the final focus quadrupoles for the $250 \times 250 \mathrm{GeV}$ and $2 \times 2$ TeV colliders.

\begin{tabular}{cccc}
\hline Collider Energy & $50 \times 50 \mathrm{GeV}$ & $250 \times 250 \mathrm{GeV}$ & $2 \times 2 \mathrm{TeV}$ \\
\hline Source Length & $20 \mathrm{~m}$ & $33 \mathrm{~m}$ & $130 \mathrm{~m}$ \\
$\mu(\mathrm{p}>1 \mathrm{GeV} / \mathrm{c})$ per electron & $9.6 \times 10^{-6}$ & $8.3 \times 10^{-5}$ & $5.4 \times 10^{-4}$ \\
Beam $\mu$ per bunch & $4 \times 10^{12}$ & $2 \times 10^{12}$ & $2 \times 10^{12}$ \\
$\mathrm{~B}-\mathrm{H} \mu$ per bunch crossing & 6100 & 17500 & 28000 \\
$\left\langle\mathrm{p}_{\mu}\right\rangle_{\text {initial }} \mathrm{GeV} / \mathrm{c}$ & 4.4 & 9.5 & 22 \\
$\mu$ 's entering calorimeter & 25 & 160 & 220 \\
$\left\langle\mathrm{p}_{\mu}\right\rangle$ in calorimeter, GeV/c & 1.8 & 6.3 & 15.4 \\
$\left\langle\mathrm{E}_{\text {dep }}\right\rangle, \mathrm{GeV}$ & 0.4 & 1.3 & 2.9 \\
Total $\mathrm{E}_{\text {dep }}$ by all $\mu$ 's & 10 & 210 & 640 \\
$\mathrm{E}_{\text {dep }}$ pedestal, GeV & 1 & 25 & 50 \\
Fluctuation in $\mathrm{E}_{\text {dep }}, \mathrm{GeV}$ & 1 & 15 & 55 \\
$\mathrm{E}_{\perp}$ pedestal, GeV & 0.5 & 15 & 15 \\
Fluctuation in $\mathrm{E}_{\perp}, \mathrm{GeV}$ & 0.5 & 8 & 40 \\
\hline
\end{tabular}

The difference in time-of-flight between signal muon path and the B-H muon path would exceed $1 \mathrm{~ns}$ which can be used to remove the background. The situation is not the same for the forward and backward calorimeters. The time difference between the path of a forward-going B-H muon reaching the forward calorimeter and path for a beam muon plus produced particle to reach the forward calorimeter is very small. The timing cut will only eliminate the $\mathrm{B}-\mathrm{H}$ muons in the backward calorimeter in this case. Thus the timing cut can only eliminate half of the B-H muon background in the forward and backward calorimeters.

\section{Pair Production}

Although coherent beam-beam electron pair production (beamsstrahlung) is small [3] incoherent pair production from processes like $\mu^{+} \mu^{-} \rightarrow \mu^{+} \mu^{-} \mathrm{e}^{+} \mathrm{e}^{-}$is significant in the $2 \times 2 \mathrm{TeV}$ collider. The cross section for such processes is estimated to be $10 \mathrm{mb}$ [4]

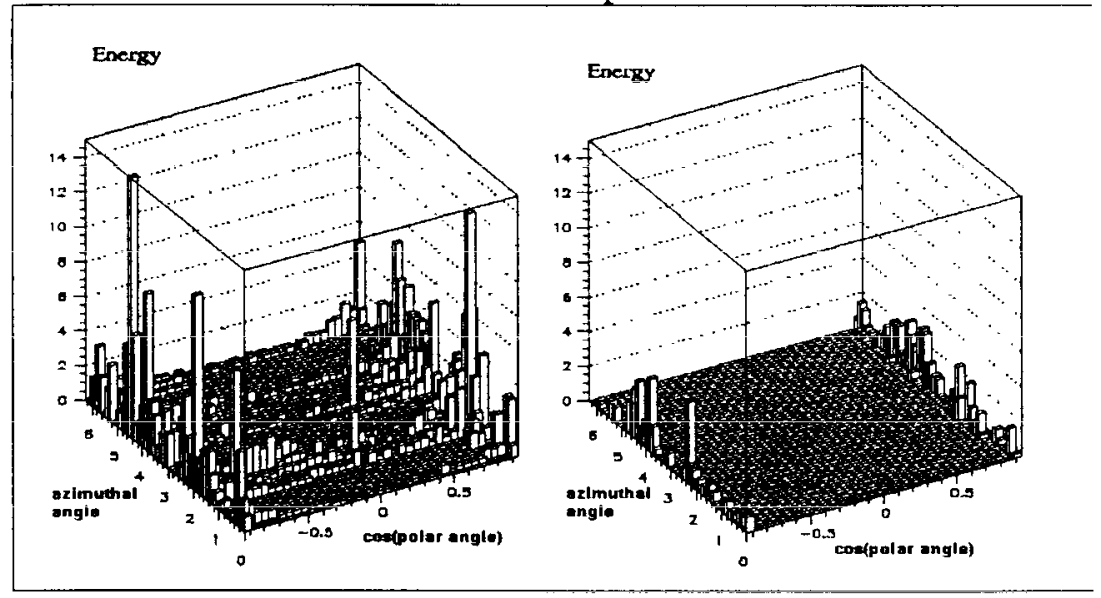


FIGURE 6. Energy deposition from Bethe-Heitler muons into a calorimeter at a $2 \times 2 \mathrm{TeV}$ muon collider. The calorimeter cells are segmented in cells with $\Delta \phi=0.1$ and $\Delta \cos \vartheta \approx 0.05$. The right-hand plot has a timing cut of 1 ns.

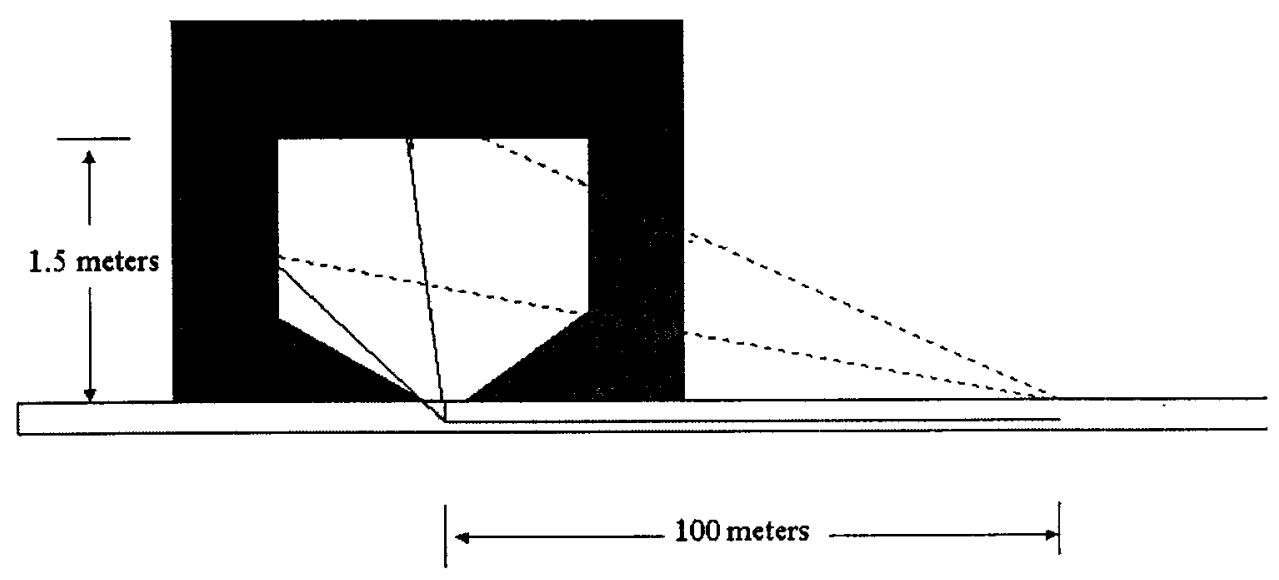

FIGURE 7. Sketch illustrating the paths of signal muons from the IP and Bethe-Heitler muons generated by an electromagnetic shower in the beam chamber wall 100 meters upstream. The difference in path length between the signal muons and the B-H muons is large enough to distinguish by a $1 \mathrm{~ns}$ difference in time-of-flight in the central calorimeter. In the forward calorimeter a 1ns difference in time-of-flight is not sufficient to be distinguished the signal muon from the B-H background. This sketch is not to scale.

which would mean approximately $3 \times 10^{4}$ electron pair per beam crossing. Although these electrons do not have significant transverse momentum initially they can be deflected into the detector by the oncoming beam. This potential background was examined with a simple tracking program to follow electrons created on the axis (the worse case) as they are deflected away from the opposing bunch. After they are past that bunch the electrons are trapped in spiral orbits in the detector magnetic field. Figure 8 shows the trajectories of the electron tracks for initial momentum in the range of 3.8 to $3000 \mathrm{MeV} / \mathrm{c}$ in a 4 tesla solenoid field. The study was done for a 2 tesla and 4 tesla detector solenoid field. In the 2 tesla case electrons with $\mathrm{P}_{\text {init }}<30 \mathrm{MeV} / \mathrm{c}$ do not make it out to the $10 \mathrm{~cm}$ detector plane, while those with $P_{\text {init }}>100 \mathrm{MeV} / \mathrm{c}$ have an initial angle that keeps them within the nose shield. Approximately $10 \%$ of the electron tracks are between these limits and pass through the detector plane at $10 \mathrm{~cm}$. This gives an electron track fluence $<10$ tracks $/ \mathrm{cm}^{2}$. For a detector plane at $5 \mathrm{~cm}$ there would be an electron track fluence of 30 tracks $/ \mathrm{cm}^{2}$. If the detector solenoid field is 4 tesla, no electron tracks reach $10 \mathrm{~cm}$ and the fluence at $5 \mathrm{~cm}$ is reduced by a factor of 2 . 


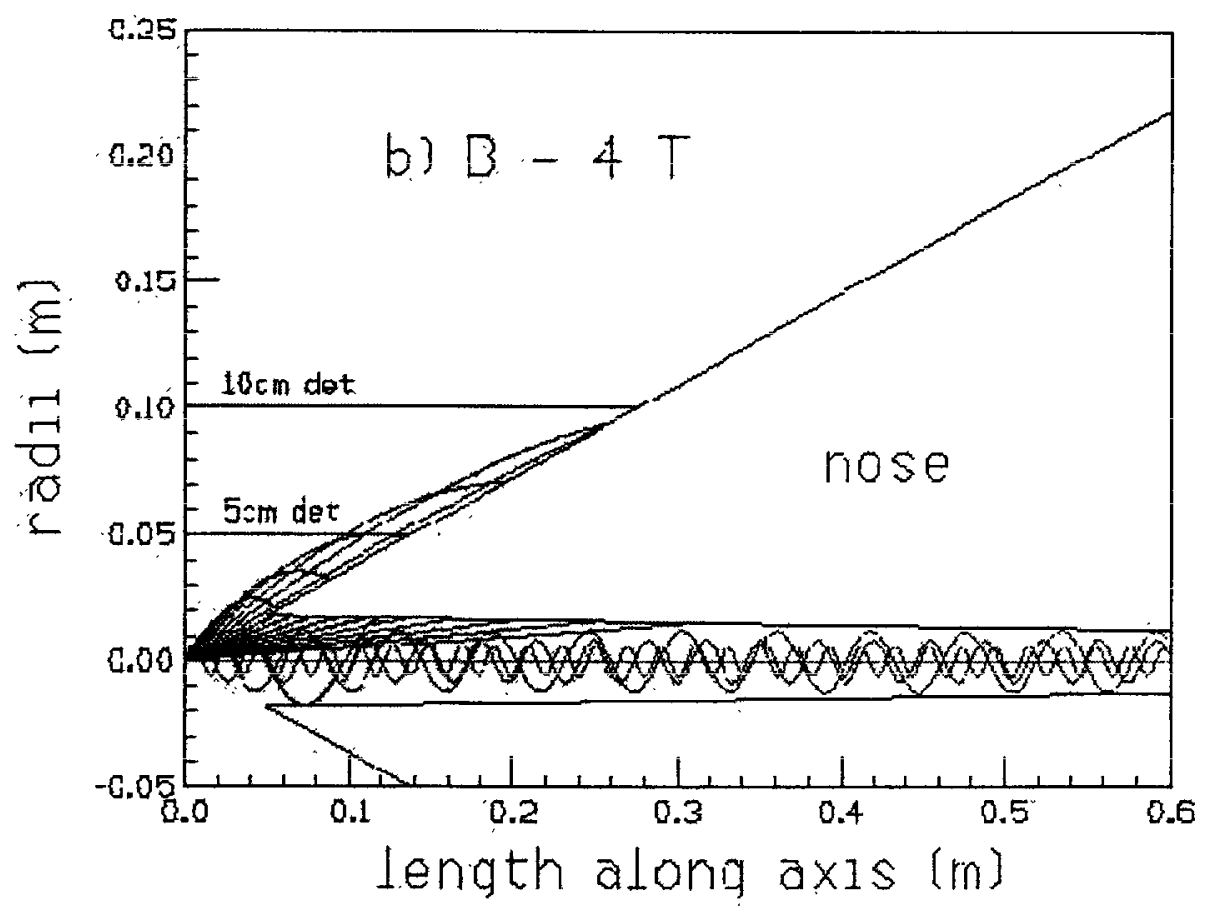

FIGURE 8. Trajectories of inelastic electron pair tracks with initial momentum between 3.8 and $3000 \mathrm{MeV} / \mathrm{c}$. These tracks are in a $4 \mathrm{~T}$ solenoid field.

\section{Beam Halo Background}

Beam halo background is the background from the tails of the beam that can get into the detector. Since muons can traverse long distances without interacting, any source of beam loss in the ring can potentially deflect some muons into detector. This is generally not as serious a problem in hadron or electron colliding rings. Since each beam has $2 \times 10^{12}$ muons, a small beam halo that could get into the detector would be very troublesome. Halo calculations are hard to do since they depend on a detailed model of the ring, the injection system and the injected beam profile. In addition imperfections in the building of the accelerator can contribute. Beam halo can have two components: (1) that which is associated to the initial filling of the ring which will die out in a smail number of turns and (2) that which is generated or regenerated in the ring and can persist throughout the fill. The former is not of as great concern since the physics data from the first few turns can be ignored.

A collimation system[5] in the ring is designed to scrape the beam at a position in the ring $180^{\circ}$ away from the IP. This system would scrape the beam at $3 \sigma$ in $x$ and $y$. Studies with very limited statistics have been performed tracking muons with $\mathrm{x}, \mathrm{x}^{\prime}, \mathrm{y}$, or $\mathrm{y}^{\prime}$ greater than $3.5,4,4.5,5$ beam $\sigma$ around a $2 \mathrm{TeV}$ lattice. In this study muons were tracked through finite sized magnets, not lumped elements. Muons were allowed pass through material with multiple scattering corrections. With samples of $200 \mu$ 's no beam halo tracks appeared in the detector region. These samples are too small to make significant conclusions. Much more work in this area needs to be done. 


\section{CONCLUSIONS}

The highest energy muon collider that physics backgrounds have been studied is the $2 \times 2 \mathrm{TeV}$ machine. It is not easy to make meaningful extrapolations to $100 \mathrm{TeV}$ com muon colliders. It is likely that certain backgrounds such as hadron production and Bethe-Heitler muons will increase with energy. It can be assumed that a very high energy muon collider in the range of $10-100 \mathrm{TeV}$ in the center of mass will not be the first machine built. One will learn much better how to estimate and control these physics backgrounds by building lower energy machines such as the $4 \mathrm{TeV}$ center of mass machine that was studied at SNOWMASS '96.

\section{ACKNOWLEDGMENTS}

This research was supported by the U. S. Department of Energy under Contract No. DE-ACO2-98CH10886.

\section{REFERENCES}

1. $\mu^{+} \mu^{-}$Collider: A Feasibility Study. BNL-52503, FNAL Conf 96/092, LBNL-38946 (1996).

2. Ankenbrandt, C. M. et al., "Status of Muon Collider Research and Development and Future Plans", Phys. Rev. Special Topics $-A B 081001$ (1999).

3. P. Chen, Beam-Beam Interactions in Muon Colliders, Nucl. Phys. B (Proc. Suppl.) 51A, 179 (1996).

4. I. Ginzburg, The $e^{+} e^{-}$Pair Production at $\mu^{+} \mu^{-}$Collider, Nucl. Phys. B (Proc. Suppl.) 51A, 186, (1996).

5. A. Drozhdin et al., Scraping Beam Halo in $\mu^{+} \mu^{-}$Colliders, AIP Conf. Proc. 441, p 242 (1998), FERMILAB-Conf-98/042. 